\title{
David Edgerton, Quoi de neuf ? Du rôle des techniques dans l'histoire globale
}

Paris, Seuil, l’Univers historique, 2013

\section{Larissa Zakharova}

\section{(2) OpenEdition \\ Journals}

\section{Édition électronique}

URL : https://journals.openedition.org/artefact/8753

DOI : $10.4000 /$ artefact. 8753

ISSN : 2606-9245

\section{Éditeur :}

Association Artefact. Techniques histoire et sciences humaines, Presses universitaires du Midi

\section{Édition imprimée}

Date de publication : 11 septembre 2014

Pagination : 226-228

ISBN : 978-2-271-08150-6

ISSN : 2273-0753

\section{Référence électronique}

Larissa Zakharova, «David Edgerton, Quoi de neuf? Du rôle des techniques dans I'histoire globale», Artefact [En ligne], 2 | 2014, mis en ligne le 11 mai 2021, consulté le 24 août 2021. URL : http:// journals.openedition.org/artefact/8753 ; DOI : https://doi.org/10.4000/artefact.8753

Ce document a été généré automatiquement le 24 août 2021.

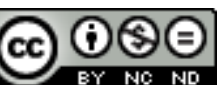

Artefact, Techniques, histoire et sciences humaines est mise à disposition selon les termes de la Licence Creative Commons Attribution - Pas d'Utilisation Commerciale - Pas de Modification 4.0 International. 


\title{
David Edgerton, Quoi de neuf? Du rôle des techniques dans l'histoire globale
}

\author{
Paris, Seuil, l'Univers historique, 2013
}

\author{
Larissa Zakharova
}

\section{RÉFÉRENCE}

David Edgerton, Quoi de neuf? Du rôle des techniques dans l'histoire globale, trad. de The Schock of the Old. Technology and Global History since 1900, Profile Books, 2006, par Christian Jeanmougin, Paris, Seuil, l'Univers historique, 2013, 320 p.

1 David Edgerton, qu'un numéro thématique des Annales consacré à l'histoire des techniques a fait connaitre au-delà du cercle des spécialistes ${ }^{1}$, cherche à montrer dans cet ouvrage de synthèse le rôle des techniques dans les phénomènes majeurs de l'histoire $\mathrm{du} \mathrm{xx}^{\mathrm{e}}$ siècle. Contrairement à la tendance historiographique qui associe la technique à l'invention et à l'innovation, Edgerton propose de repenser le temps technologique en examinant la durée de vie des techniques qui se manifeste à travers leurs usages. Cette approche est, d'après lui, la seule à pouvoir s'inscrire dans un projet d'histoire globale, car l'histoire centrée sur les innovations concernerait très peu de pays dans le monde, excluant en particulier le continent africain.

2 L'ouvrage est structuré autour de huit chapitres thématiques (l'importance, le temps, la production, l'entretien, les nations, la guerre, la mise à mort et l'invention) qui examinent ce que l'auteur identifie à des idées reçues, mais qui sont en réalité le plus souvent des idées datées (par ailleurs battues en brèche par l'historiographie récente). On peut distinguer trois thèses majeures dans cet ouvrage.

La première insiste sur les chevauchements du temps technologique. Celui-ci n'est pas linéaire: reculs, retours en arrière sont fréquents. C'est la longévité des techniques considérées comme « archaïques » qui prouve que les nouveautés ne remplacent jamais entièrement et définitivement les techniques anciennes. Le retour de l'ancien peut se manifester à travers la valorisation de techniques "rétro", comme le montre 
l'agriculture biologique. La machine à coudre est une technique dont la longévité est extraordinaire. L'ancien côtoie le nouveau : le condom ne disparaît pas avec la diffusion de la pilule contraceptive à partir des années 1960 et revient même en force dans les années 1980 en raison de l'épidémie du SIDA. De la même façon, la guerre au xx siècle est restée une affaire de fusils, de tanks et d'avions, plutôt que de gaz, de radars et d'armes atomiques. Les armes éprouvées par le temps et dont l'usage est établi, s'avèrent les plus meurtrières. C'est l'artillerie lourde qui a provoqué le plus de dégâts et de morts lors des deux guerres mondiales. La deuxième place est occupée par les armes légères : la Kalachnikov a rencontré un "succès » inégalé tant par le nombre d'exemplaires que par la longévité. Les armes les plus puissantes de la Grande Guerre, à l'instar des cuirassés dreadnought, sont celles qui sont les moins utilisées. Inventée aux États-Unis dans l'entre-deux-guerres pour exécuter les condamnés à mort, la chambre à gaz est ensuite utilisée par l'Allemagne nazie à des fins génocidaires. Lors de la Seconde Guerre mondiale, elles tuent autant que les armes légères. Les techniques de mise à mort révèlent donc l'interaction entre l'ancien et le nouveau.

4 Les chevauchements du temps technologique renvoient à des chronologies décalées d'un pays à l'autre. Le rythme d'adoption de nouvelles techniques dépend du niveau de vie. Quand un pays s'enrichit, une frange plus importante de la population s'équipe de techniques déjà présentes sur le marché. Mais, comme, entretemps, ce pays a continué à utiliser des techniques "anciennes" qui ont subsisté malgré l'introduction des nouveautés, la modernisation s'opère de façon particulière. Aussi, l'idée d'innovation globale qui entraîne une croissance économique globale, est une utopie qui ne résiste pas à l'analyse à l'échelle nationale.

5 Les décalages chronologiques et les inégalités entre les régions du monde doivent être pris en compte lorsqu'on pense à ce qu'est une innovation. C'est la deuxième grande thèse de ce livre: l'innovation est davantage marquée par la continuité que par la rupture. Si l'on suit la différenciation opérée par David Edgerton, il y a beaucoup plus d'imitations que d'inventions dans le monde: chaque imitation demande de l'inventivité. Les réparations et l'entretien qui remodèlent souvent des techniques, sont aussi une source de l'innovation. La pénurie de pièces de rechange est à l'origine de techniques nouvelles. Les techniques créoles sont des dérivés locaux de quelque chose originaire d'ailleurs. Ces hybridations donnent une nouvelle vie aux techniques importées dans les pays pauvres, dont les usages sont ainsi prolongés. La réparation d'une voiture à l'aide des moyens du bord et du bricolage s'appuie sur des savoir-faire originaux. Le cyclo-pousse dérivé des vélos coexiste avec les automobiles. Les habitations des bidonvilles utilisent des produits de l'industrie locale et globale. Les dépenses pour l'entretien et la réparation sont en moyenne corrélées aux investissements dans les innovations. Plus le pays est pauvre, moins l'entretien et la réparation sont coûteux et plus la vie des techniques est longue. Dans les pays riches, le coût élevé de l'entretien et de la réparation explique la tendance à acheter du neuf. L'entretien amène à la fabrication de composantes puis des techniques entières. Dans les années 1950, la réparation des postes de radio au Japon conduit à la fabrication de téléviseurs dans les mêmes ateliers. La science et les universités n'ont donc pas le monopole de l'invention. La grande majorité des innovations provient de l'industrie. En même temps, les techniques qui sont souvent considérées comme des vecteurs de la globalisation - l'aviation, la radio, l'internet - sont initialement des techniques militaires. L'armée produit donc des innovations majeures, ce qui contredit l'idée datée 
selon laquelle les techniques militaires ne sont que des déclinaisons des techniques civiles.

6 Enfin, la troisième thèse concerne les choix et les retombées des techniques. Elle s'oppose au déterminisme technologique. Un nombre important des techniques échoue. Pour cette raison, elles ne figurent ni dans les histoires conventionnelles des techniques, ni dans les musées spécialisés. Les brevets ne sont pas des indicateurs fiables de l'inventivité, car ils ne disent rien des techniques qui «ont échoué » et de celles qui ne sont pas brevetées. Le brevet ne garantit ni la longue vie, ni le succès de l'invention. Les utilisateurs ont toujours le choix d'adopter ou non telle ou telle technique. La résistance à certaines techniques ne peut pas être assimilée au conservatisme. Cette résistance a du sens entre autres parce que, du point de vue économique, nombre de techniques sont produites à perte, malgré les déclarations de leurs promoteurs (par exemple, les réacteurs nucléaires). Comment mesurer temporellement l'impact d'une technique? Économiquement, quantitativement, culturellement? Pour David Edgerton, ce sont les usages à long terme qui permettent de rendre compte des retombées des techniques. Il s'agit de la rentabilité dynamique, qui se distingue de la rentabilité statique par la prise en compte de la durée. D. Edgerton estime alors qu'un moyen de mesurer l'importance d'une technique consiste à pratiquer l'histoire contrefactuelle (calculer les coûts et les bénéfices dans un monde qui ignorerait la technique en question).

7 L'innovation ne conduit pas automatiquement à la croissance, bien que le financement de la recherche et du développement par l'État soit destiné à assurer la performance économique. Ces théories de la croissance endogène et, plus généralement, le technonationalisme qui consiste à célébrer (et à étudier) les inventeurs nationaux, représentent une posture erronée, car les techniques ne connaissent pas de frontières. Même lors des périodes à forte tendance autarcique, les produits et les experts traversent les frontières des États. Le décalage entre l'invention et la croissance passe par l'usage: les pays qui comptent le plus d'inventions ne sont pas forcement les utilisateurs les plus actifs des innovations. Dans les années 1980, l'Italie dépasse la Grande-Bretagne par le taux de la croissance économique malgré le faible investissement dans la recherche et le développement. Les États-Unis sont la seule exception, car les innovations nationales $\mathrm{y}$ ont un impact économique très fort. Le problème est que cette corrélation a été généralisée à l'ensemble des pays.

8 Avec l'argument de la régression technologique à l'appui (le démantèlement des navires en Inde se fait avec des techniques de plus en plus rudimentaires), le livre propose en guise de conclusion l'idée selon laquelle le passé a été plus inventif que le présent. Cette affirmation est surprenante pour un livre qui insiste tant sur l'importance de la durée. En outre, le livre présente des défauts liés à un raisonnement circulaire : la réfutation d'une idée laisse progressivement la place aux arguments en faveur de cette même idée. Cependant, le mérite de cet ouvrage est de placer au cœur des analyses les usages des techniques non seulement célèbres (généralement associées à la modernité), mais aussi banales et quotidiennes et qui s'avèrent tout aussi cruciales pour repenser l'histoire du $\mathrm{xx}^{\mathrm{e}}$ siècle. 


\section{NOTES}

1. David Edgerton, «De l'innovation aux usages. Dix thèses éclectiques sur l'histoire des techniques ", Annales. Histoire, Sciences Sociales, n 4-5, 1998, p. 815-837.

\section{AUTEURS}

LARISSA ZAKHAROVA

EHESS-CERCEC 